\title{
Epidemiology of human papillomavirus genotypes in pregnant Japanese women
}

\author{
Kentaro Yamasaki ${ }^{1}$, Kiyonori Miura ${ }^{1}$, Takako Shimada $^{1}$, Shoko Miura ${ }^{1}$, Shuhei Abe ${ }^{1}$, Makoto Murakami $^{2}$, \\ Tetsuro Sameshima ${ }^{3}$, Akira Fujishita ${ }^{4}$, Kouhei Kotera ${ }^{5}$, Akira Kinoshita ${ }^{6}$, Koh-ichiro Yoshiura ${ }^{6}$ and \\ Hideaki Masuzaki ${ }^{1}$
}

To investigate the pre-vaccination epidemiology of genital human papillomavirus (HPV) infections and genotypes in pregnant Japanese women, we performed Pap smear tests and HPV genotype testing in patients attending Nagasaki University Hospital and collaborating hospitals from August 2007 to July 2010. Serial uterine cervical specimens were obtained from 151 pregnant women. The HPV test was positive on the first visit in 54 women (35.8\%; 54/151, average age 30). A total of 49 women $(32.5 \% ; 49 / 151)$ were infected by at least one high-risk HPV and 5 women were infected by only low-risk HPV. The three most prevalent high-risk HPV genotypes were HPV 52 (31.5\%; 17/54), HPV 16 (29.6\%; 16/51) and HPV 31 (13.0\%; 7/51). The HPV infection pattern (negative, single infection and multiple infection) differed significantly according to the pregnancy trimester $\left(\chi^{2}\right.$-test; $\boldsymbol{P}<0.01$ (Pearson)). Among HPV-infected pregnant Japanese women, HPV52 was the most common genotype. The second most common genotype was HPV16, and these two genotypes accounted for $\sim 60 \%$ of HPV-positive pregnant women. Infection with multiple HPV genotypes was observed more frequently in the first trimester of pregnancy and the pattern of infection changed significantly depending on pregnancy stage.

Journal of Human Genetics (2011) 56, 313-315; doi:10.1038/jhg.2011.11; published online 10 February 2011

Keywords: epidemiology; genotype; HPV; infection; pregnancy; uterine cervical neoplasia

\section{INTRODUCTION}

Persistent infections with human papillomavirus (HPV) are recognized as a major cause of cervical cancer. Genital infections with HPV are very common, and these infections are transmitted by sexual contact. ${ }^{1}$ In most cases, HPV infections disappear naturally in a relatively short time period and carry only a small risk of disease development; ${ }^{2-4}$ however, some investigators believe that pregnancy affects the host immune system; for example, pregnancy is believed to reduce seroreactivity against HPV infection. ${ }^{5}$ Estrogen and progesterone have been shown to activate the upstream regulatory region of HPV18 (see ref. 6). Thereby altering HPV clearance rates compared with non-pregnant women. During persistent infection, important factors of disease susceptibility include HPV genotypes and viral characteristics, such as the distribution of each type in the population and the evasive ability against the host's immune system. It is not clear how persistent infection relates to the host, such as how the host immune system reacts against a specific HPV genotype and how sexual behavior in pregnant women affects infection rates. In particular, there are very few data detailing HPV genotype prevalence in pregnant Japanese women. To determine the distribution of HPV infections in pregnant Japanese women, we performed HPV genotype testing.

\section{MATERIALS AND METHODS}

Study population and sample collection

Cytology and HPV DNA test samples were collected by six hospitals that collaborated with Nagasaki University Hospital from August 2007 to July 2010. All pregnant women whose first visit during pregnancy was to one of our collaborating hospitals were invited to join the study. An obstetrics doctor explained the study aim, procedures and complications. We did not inform participants of the HPV genotyping results. Exclusion criteria were patients who had previously received therapeutic surgery or who had histologically confirmed non-squamous neoplasms. We registered 151 pregnant women having a routine Pap test screen for the study. Specimens were taken with a Cervex brush (Rovers Medical Devices, Oss, Netherlands) and suspended in $10 \mathrm{ml}$ of SurePath preservative fluid (Becton Dickinson, Franklin Lakes, NJ, USA). We used sample from the same vial for cytology and for HPV genotype testing. Cervical specimens for HPV genotyping

${ }^{1}$ Department of Obstetrics and Gynecology, School of Medicine, Nagasaki University, Nagasaki, Japan; 2Department of Obstetrics and Gynecology, Sasebo Municipal Hospital, Sasebo, Japan; ${ }^{3}$ Department of Obstetrics and Gynecology, the Japanese Red Cross Nagasaki Genbaku Hospital, Nagasaki, Japan; ${ }^{4}$ Department of Obstetrics and Gynecology, Saiseikai Hospital, Nagasaki, Japan; ${ }^{5}$ Department of Obstetrics and Gynecology, Nagasaki Municipal Hospital, Nagasaki, Japan and ${ }^{6}$ Department of Human Genetics, School of Medicine, Nagasaki University, Nagasaki, Japan

Correspondence: Dr K Miura, Department of Obstetrics and Gynecology, School of Medicine, Nagasaki University, 1-7-1 Sakamoto, Nagasaki 852-8501, Japan. E-mail: kiyonori@nagasaki-u.ac.jp

Received 30 November 2010; revised 26 December 2010; accepted 12 January 2011; published online 10 February 2011 
were taken at each visit from participants who received regular follow-up examinations.

The study protocol was approved by the ethical review board of Nagasaki University and of the other hospitals involved. All women were informed of the purpose of the study and gave their consent.

\section{HPV genotyping test}

Genotyping of HPV DNA in the SurePath preservative fluid was performed using the Linear Array HPV Genotyping Test kit (Roche Diagnostics, Indianapolis, IN, USA). The kit uses PGMY09/PGMY11 primers ${ }^{7}$ to amplify the L1 conserved region. Following PCR amplification, hybridization of the HPV amplicon was performed using an array of oligonucleotide probes that allowed independent identification of individual HPV genotypes. This kit can detect $37 \mathrm{HPV}$ genotypes $(6,11,16,18,26,31,33,35,39,40,42,45,51,52,53$, $54,55,56,58,59,61,62,64,66,67,68,69,70,71,72,73$ (MM9), 81, 82 (MM4), 83 (MM7), 84 (MM8), IS39 and CP6108 (89)). For consistency with previous studies, we considered 16 HPV genotypes $(16,18,31,33,35,45,51$, $52,53,56,58,59,66,68,73$ and 82 ) as high-risk genotypes that are related to cervical cancer. ${ }^{8-10}$

\section{RESULTS}

A total of 151 women were enrolled in the study. In all, 79 women were in the first trimester of their pregnancy, 18 were in the second trimester, 51 were in the third trimester and 3 were postpartum. Fiftyfour women $(35.8 \% ; 54 / 151)$, with an average age of 30 , were positive

Table 1 Prevalence of HPV infection in pregnant Japanese women

\begin{tabular}{|c|c|c|c|c|}
\hline & \multicolumn{3}{|c|}{ Trimester } & \multirow[b]{2}{*}{ Total $(\%)^{a}$} \\
\hline & $1 s t$ & 2nd & $3 r d$ & \\
\hline$n$ & 79 & 18 & 51 & 148 \\
\hline HPV negative & 48 & 7 & 39 & 94 \\
\hline Single infection & 17 & 9 & 10 & $36(66.7)$ \\
\hline Multiple infection & 14 & 2 & 2 & 18 (33.3) \\
\hline \multicolumn{4}{|l|}{ HPV type } & \\
\hline 16 & 7 & 6 & 3 & $16(29.6)$ \\
\hline 18 & 2 & 2 & 1 & $5(9.3)$ \\
\hline 31 & 5 & 0 & 2 & $7(13.0)$ \\
\hline 33 & 3 & 0 & 0 & $3(5.6)$ \\
\hline 51 & 2 & 0 & 0 & $2(3.7)$ \\
\hline 52 & 14 & 2 & 1 & $17(31.5)$ \\
\hline 53 & 1 & 0 & 0 & $1(1.9)$ \\
\hline 56 & 3 & 0 & 0 & $3(5.6)$ \\
\hline 58 & 2 & 1 & 2 & $5(9.3)$ \\
\hline 68 & 2 & 0 & 0 & $2(3.7)$ \\
\hline 82 & 1 & 0 & 1 & $2(3.7)$ \\
\hline
\end{tabular}

\begin{tabular}{|c|c|c|c|c|}
\hline 6 & 2 & 1 & 0 & $3(5.6)$ \\
\hline 39 & 1 & 0 & 1 & $2(3.7)$ \\
\hline 42 & 0 & 0 & 1 & 1 (1.9) \\
\hline 54 & 1 & 1 & 0 & $2(3.7)$ \\
\hline 61 & 3 & 0 & 1 & $4(7.4)$ \\
\hline 71 & 0 & 0 & 1 & 1 (1.9) \\
\hline 84 & 0 & 0 & 2 & $2(3.7)$ \\
\hline CP6108 (89) & 2 & 0 & 0 & $2(3.7)$ \\
\hline
\end{tabular}

Abbreviation: HPV, human papillomavirus.

aThe denominator was the number of HPV-positive women. for HPV on their first hospital visit. Forty-nine women (32.5\%; 49/ 151) were infected by at least one high-risk HPV and five women were infected by only low-risk HPV. The three most prevalent high-risk HPV genotypes were HPV 52 (31.5\%; 17/54), HPV 16 (29.6\%; 16/51) and HPV $31(13.0 \%$; 7/51) (Table 1). The HPV infection pattern (negative, single infection and multiple infection) was significantly different depending on the trimester of the pregnancy $\left(\chi^{2}\right.$-test; $P<0.01$ (Pearson)).

\section{DISCUSSION}

The influence of pregnancy on the natural course of HPV infection is not understood. Several reports show that the prevalence of HPV infection in pregnant women is variable at $10-60 \%$, depending on age, region and HPV detection methods. ${ }^{11-14}$ The HPV infection rate in pregnant women in this report was $35 \%$. There are few reports of HPV infection rates in pregnant Japanese women. One report by Takakuwa et al. ${ }^{15}$ showed the prevalence of HPV in pregnant Japanese women to be $12.5 \%$. The difference between this report and our results may be because of the detection method used, PCR-reverse hybridization (our study) and PCR-restriction fragment length polymorphism (Takakuwa et al.), and the number of detectable HPV genotypes.

Several other studies ${ }^{16,17}$ show the clearance of HPV infection may accelerate in the third trimester and postpartum. In our study, the HPV infection pattern differed significantly according to the pregnancy trimester. In particular, in the first trimester, multiple HPV infection was observed more often than in the later periods. This observation may be explained by changes in sexual behavior and/or by immunological factors. Pregnant women tend to be less sexually active and their immune response against HPV is reduced. The accelerated clearance of HPV may be due to the host immune system normalizing during the third trimester. ${ }^{18}$

Our results showed that HPV 16 and HPV 52 were the two most common genotypes among pregnant Japanese women. Our data among 154 non-pregnant Japanese women also showed that HPV 16 and HPV 52 were the two most common genotype. The prevalence of HPV genotype in Japanese pregnant women may not show pregnant-specific features. Other reports using HPV genotyping tests also showed that HPV 52 was a more common genotype among Japanese individuals who had either normal cytology or cervical neoplastic lesions compared with individuals in other countries. $^{10,19,20}$

In Japan, a commercial cervical cancer vaccine finally became available after December 2009. This study has some limitations because we obtained data from pregnant women; however, our data from pre-vaccination women on the distribution of genital HPV infections in the region where HPV 52 is more prevalent are important for the understanding of the cross-reactivity of a bivalent HPV vaccine. ${ }^{17,21}$

\section{ACKNOWLEDGEMENTS}

K Yamasaki was supported by the Nagasaki University President's Fund Grant. K Miura, S Miura and H Masuzaki were supported by a Grant-in-Aid for Young Scientists (B) (No. 21791567, No. 22791535) and for Scientific Research (C) (No. 22591827) from the Ministry of Education, Culture, Sports, Science and Technology of Japan. K Miura was was supported by The Grant of National Center for Child Health and Development (20C-1), Japan. K Yoshiura was supported by Grants-in-Aid for Scientific Research from the Ministry of Health, Labour and Welfare, Japan. 
1 Shimada, T., Miyashita, M., Miura, S., Nakayama, D., Miura, K., Fukuda, M. et al. Genital human papilloma virus infection in mentally-institutionalized virgins. Gynecol. Oncol. 106, 488-489 (2007).

2 Moscicki, A. B., Palefsky, J., Smith, G., Siboshski, S. \& Schoolnik, G. Variability of human papillomavirus DNA testing in a longitudinal cohort of young women. Obstet. Gynecol. 82(Part 1), 578-585 (1993).

3 Woodman, C. B., Collins, S., Winter, H., Bailey, A., Ellis, J., Prior, P. et al. Natural history of cervical human papillomavirus infection in young women: a longitudinal cohort study. Lancet. 357, 1831-1836 (2001).

4 Ho, G. Y., Bierman, R., Beardsley, L., Chang, C. J. \& Burk, R. D. Natural history of cervicovaginal papillomavirus infection in young women. N. Engl. J. Med. 338, 423-428 (1998).

5 Sethi, S., Muller, M., Schneider, A., Blettner, M., Smith, E., Turek, L. et al. Serologic response to the $E 4, E 6$, and $E 7$ proteins of human papillomavirus type 16 in pregnant women. Am. J. Obstet. Gynecol. 178, 360-364 (1998).

6 Michelin, D., Gissmann, L., Street, D., Potkul, R. K., Fisher, S., Kaufmann, A. M. et al. Regulation of human papillomavirus type 18 in vivo: effects of estrogen and progesterone in transgenic mice. Gynecol. Oncol. 66, 202-208 (1997).

7 Gravitt, P. E., Peyton, C. L., Alessi, T. Q., Wheeler, C. M., Coutlee, F., Hildesheim, A. et al. Improved amplification of genital human papillomaviruses. J. Clin. Microbiol. 38, 357-361 (2000).

8 Walboomers, J. M., Jacobs, M. V., Manos, M. M., Bosch, F. X., Kummer, J. A., Shah, K. V. et al. Human papillomavirus is a necessary cause of invasive cervical cancer worldwide. J. Pathol. 189, 12-19 (1999).

9 Munoz, N., Bosch, F. X., de Sanjose, S., Herrero, R., Castellsague, X., Shah, K. V. et al. Epidemiologic classification of human papillomavirus types associated with cervical cancer. N. Engl. J. Med. 348, 518-527 (2003).

10 Asato, T., Maehama, T., Nagai, Y., Kanazawa, K., Uezato, H. \& Kariya, K.-I.Alarge Casecontrol study of cervical cancer risk associated with human papillomavirus infection in Japan, by nucleotide sequencing-based genotyping. J. Infect. Dis. 189, 1829-1832 (2004).

11 Kemp, E. A., Hakenewerth, A. M., Laurent, S. L., Gravitt, P. E. \& Stoerker, J. Human papillomavirus prevalence in pregnancy. Obstet. Gynecol. 79, 649-656 (1992).
12 Banura, C., Franceschi, S., van Doorn, L. J., Arslan, A., Kleter, B., Wabwire-Mangen, F. et al. Prevalence, incidence and clearance of human papillomavirus infection among young primiparous pregnant women in Kampala, Uganda. Int. J. Cancer. 123, 2180-2187 (2008).

13 Chan, P. K., Chang, A. R., Tam, W. H., Cheung, J. L. \& Cheng, A. F Prevalence and genotype distribution of cervical human papillomavirus infection: comparison between pregnant women and non-pregnant controls. J. Med. Virol. 67, 583-588 (2002).

14 Morrison, E. A., Gammon, M. D., Goldberg, G. L., Vermund, S. H. \& Burk, R. D. Pregnancy and cervical infection with human papillomaviruses. Int. J. Gynaecol. Obstet. 54, 125-130 (1996)

15 Takakuwa, K., Mitsui, T., Iwashita, M., Kobayashi, I., Suzuki, A., Oda, T. et al. Studies on the prevalence of human papillomavirus in pregnant women in Japan. J. Perinat. Med. 34, 77-79 (2006).

16 Fife, K. H., Katz, B. P., Brizendine, E. J. \& Brown, D. R. Cervical human papillomavirus deoxyribonucleic acid persists throughout pregnancy and decreases in the postpartum period. Am. J. Obstet. Gynecol. 180, 1110-1114 (1999).

17 Rando, R. F., Lindheim, S., Hasty, L., Sedlacek, T. V., Woodland, M. \& Eder, C. Increased frequency of detection of human papillomavirus deoxyribonucleic acid in exfoliated cervical cells during pregnancy. Am. J. Obstet. Gynecol. 161, 50-55 (1989).

18 Nobbenhuis, M. A., Helmerhorst, T. J., van den Brule, A. J., Rozendaal, L., Bezemer, P. D., Voorhorst, F. J. et al. High-risk human papillomavirus clearance in pregnant women: trends for lower clearance during pregnancy with a catch-up postpartum. Br. J. Cancer. 87, 75-80 (2002).

19 Miura, S., Matsumoto, K., Oki, A., Satoh, T., Tsunoda, H., Yasugi, T. et al. Do we need a different strategy for HPV screening and vaccination in East Asia? Int. J. Cancer. 119, 2713-2715 (2006).

20 Inoue, M., Sakaguchi, J., Sasagawa, T. \& Tango, M. The evaluation of human papillomavirus DNA testing in primary screening for cervical lesions in a large Japanese population. Int. J. Gynecol. Cancer. 16, 1007-1013 (2006).

21 Paavonen, J., Naud, P., Salmeron, J., Wheeler, C. M., Chow, S. N., Apter, D. et al. Efficacy of human papillomavirus (HPV)-16/18 ASO4-adjuvanted vaccine against cervical infection and precancer caused by oncogenic HPV types (PATRICIA): final analysis of a double-blind, randomised study in young women. Lancet. 374, 301-314 (2009). 